\title{
ESTIMATIVA DO FLUXO DE CALOR NO SOLO A PARTIR DA TEMPERATURA DO SOLO EM SÃO PAULO, SP
}

\author{
Frederico Luiz FUNARI
}

Augusto José PEREIRA FILHO

\begin{abstract}
RESUMO
O fluxo de calor no solo é um dos componentes do balanço de energia, que, junto com calor latente e sensível e os processos biológicos, é resultado da partição da energia líquida disponível. Esse índice geralmente é obtido a partir de medições com fluxímetros, termômetros de solo, ou ainda, estimado por satélites ambientais, segundo metodologias específicas. Neste trabalho, utilizaram-se medições de temperatura do solo a 0 e $20 \mathrm{~cm}$ de profundidade para calcular o fluxo de calor no solo por meio do método de DECICO (1967). As medições foram realizadas em uma área de Latossolo Vermelho-Amarelo no Parque Estadual Fontes do Ipiranga (PEFI), importante remanescente preservada da Mata Atlântica na Região Metropolitana de São Paulo, que dispõe de uma importante série histórica de dados climáticos. Os resultados indicam que o fluxo de calor médio é de 3,69 $\mathrm{W} \mathrm{m}^{-2}$ ou 2,83\% da energia líquida disponível, semelhante a áreas de Mata Atlântica e Floresta Amazônica.
\end{abstract}

Palavras-chave: Fluxo de calor no solo; Temperatura do solo; Balanço de energia; Parque Estadual Fontes do Ipiranga; Mata Atlântica.

\section{ABSTRACT}

ESTIMATION OF THE SOIL HEAT FLUX FROM THE TEMPERATURE OF THE SOIL IN SÃO PAULO, SP. The soil heat flux is one of the components of the energy balance and, together with the latent and sensible heat fluxes and biological processes, is the result of the partition of the available net energy balance. This parameter is usually measured with soil flux meters or soil temperature probes, or is estimated by environmental satellites using specific methods. In this study, soil temperature measurements on the surface and at $20 \mathrm{~cm}$ depth were used to estimate the soil heat flux with the DECICO (1967) method. The measurements were carried out in an area of Yellow-Red Latosol located in the State Park of Fontes do Ipiranga, which is an important remnant of the Atlantic Forest in the São Paulo Metropolitan Area and where an important historical series of climatic data was collected. The results indicate that the average heat flux is $3.69 \mathrm{~W} \mathrm{~m}^{-2}$ or $2.83 \%$ of the net available energy, which is similar to the average heat flux in areas of the Atlantic and Amazon forest.

Keywords: Soil heat flux; Soil temperature; Energy balance; State Park of Fontes do Ipiranga; Atlantic Forest.

\section{INTRODUÇÃO}

O balanço de energia radiante ou radiação líquida $(R n)$ é o saldo de radiação no ambiente. É a fonte de energia disponível para os processos de evapotranspiração (calor latente), aquecimento do ar atmosférico (calor sensível) e do solo (fluxo de calor no solo $-G$ ), além dos processos de fotossíntese, respiração e aquecimento dos vegetais.

Os processos físicos, químicos e biológicos no solo dependem do fluxo de calor em seu interior. Deste modo, a quantificação de $G$ é de importância, pois representa a entrada e saída de energia, que contribui para o aumento e redução 
nos fluxos de calor latente e sensível e altera as taxas de evapotranspiração.

Há diferentes métodos para obtenção do fluxo de calor no solo $(G)$. Diversos estudos foram realizados utilizando-se de fluxímetros para medição em diferentes climas, solos e tipos de cultura (GALVANI et al. 2001, PINTO 2003, LEÃO et al. 2007, SILVA et al. 2007, ANDRÉ et al. 2010, SILVA et al. 2010, PEREIRA et al. 2011, entre outros). Fluxímetros são sensores com termopilhas instalados no solo em profundidades entre 2 e $20 \mathrm{~cm}$, com acurácia de $3 \%$. Seu uso requer um registrador digital específico (datalogger), que é um equipamento de custo geralmente elevado.

Por outro lado, MACHADO et al. (2010), LOPES et al. (2011) e ANDRADE et al. (2009) estimaram $G$ a partir de dados do satélite LANDSAT com os algoritmos SEBAL e MODIS.

SOARES (2013) comparou resultados de $G$ para solo Latossolo Amarelo em uma cultura de mamona em Areia, PB, com medições de fluxímetros e estimativa a partir de termistores (para a medição de temperatura do solo), obtendo coeficiente de correlação de 0,957 entre tais medidas.

Os termistores são utilizados para corrigir os dados dos fluxímetros, pois estes últimos não podem ficar sob o efeito da radiação direta; por este motivo, eles são instalados em profundidades de 2 a $10 \mathrm{~cm}$, dependendo do objetivo do estudo (BORGES 2006).

DECICO (1974) obteve $G$ a partir da equação de calor (FOURIER 1822) e do método desenvolvido por DECICO (1967), que considera a densidade do fluxo de calor, condutividade térmica do solo, umidade volumétrica e a área através da qual se estuda a variação desse fluxo.

A metodologia de DECICO (1967) é útil, uma vez que fluxímetros tem custo elevado na maioria dos casos. Já os dados obtidos por satélite têm como obstáculo as condições do céu sobre a região estudada que deve ser claro, de preferência, o que não é fácil pelo fato do Brasil estar em uma faixa de latitude tropical, e também a relativa baixa resolução temporal e/ou espacial das imagens de sensoriamento remoto.

Em trabalho realizado para a cidade de São Paulo, FUNARI \& PEREIRA FILHO (2014) determinaram a partição do Balanço de Energia para o Parque Estadual das Fontes do Ipiranga (PEFI), um importante remanescente da Mata Atlântica inserida na Região Metropolitana de São Paulo (RMSP). No entanto, os resultados deste estudo foram obtidos a partir de apenas 12 dias e não se discutiu a magnitude, as variações sazonais e a importância de $G$ em relação à $R n$.

O objetivo deste trabalho é estimar $G$ a partir da metodologia de DECICO (1967) em uma área de Latossolo Vermelho-Amarelo no Parque Estadual Fontes do Ipiranga (PEFI). Trata-se da mesma área estudada por FUNARI \& PEREIRA FILHO (2014), porém utilizando uma série histórica mais ampla (2011 a 2016). Além disso, discute-se as variações sazonais de $G$ e sua importância em relação à $R n$. Ressalta-se o interesse desse trabalho, uma vez que há poucos estudos sobre $G$ em áreas de Mata Atlântica.

\section{2 ÁREA DE ESTUDO}

O estudo foi realizado no Parque Estadual Fontes do Ipiranga (PEFI), onde estão instaladas as estações meteorológicas do IAG-USP (Estação Meteorológica Convencional - EMC e Estação Meteorológica Automática - EMA), que fornecem uma série de dados hidrometeorológicos com consistência e frequência, como temperatura do solo $(T S)$ e o saldo de radiação $(R n)$, além de outras variáveis climáticas. Ambas estações estão instaladas no mesmo cercado meteorológico, que se localiza nas coordenadas $23^{\circ} 39^{\prime} 04^{\prime \prime} \mathrm{S}$ e $46^{\circ} 37^{\prime} 21^{\prime \prime} \mathrm{O}$, na altitude de $802 \mathrm{~m}$. As estações meteorológicas estão aproximadamente no centro geométrico do Parque Estadual das Fontes do Ipiranga, na região sul da cidade de São Paulo (Figura 1).

O PEFI é uma reserva de Mata Atlântica inserida na RMSP em meio à densa área urbana da cidade de São Paulo (Figura 1). A sua localização, próxima da costa oriental do continente Sul-Americano e junto ao Trópico de Capricórnio, implica em uma realidade climática de transição, entre os climas tropicais, com período seco definido, e aqueles subtropicais, permanentemente úmidos do Brasil (TARIFA \& ARMANI 2001). O clima da cidade de São Paulo é classificado como tropical úmido de altitude (TARIFA \& ARMANI 2001), com um regime pluviométrico de concentração de chuvas na primavera e verão, com redução no outono e inverno, apresentando um total anual pluviométrico médio de 1402,8 mm (1933-2014) (IAG 2015). A radiação global média anual (1961-2007) é de $177,0 \mathrm{~W} \mathrm{~m}^{-2} \mathrm{e}$ a insolação anual, de $1879 \mathrm{~h}$ ano ${ }^{-1}$. O saldo do balanço de radiação tem uma média anual de $114 \mathrm{~W} \mathrm{~m}^{-2}$ (FUNARI 1983). 

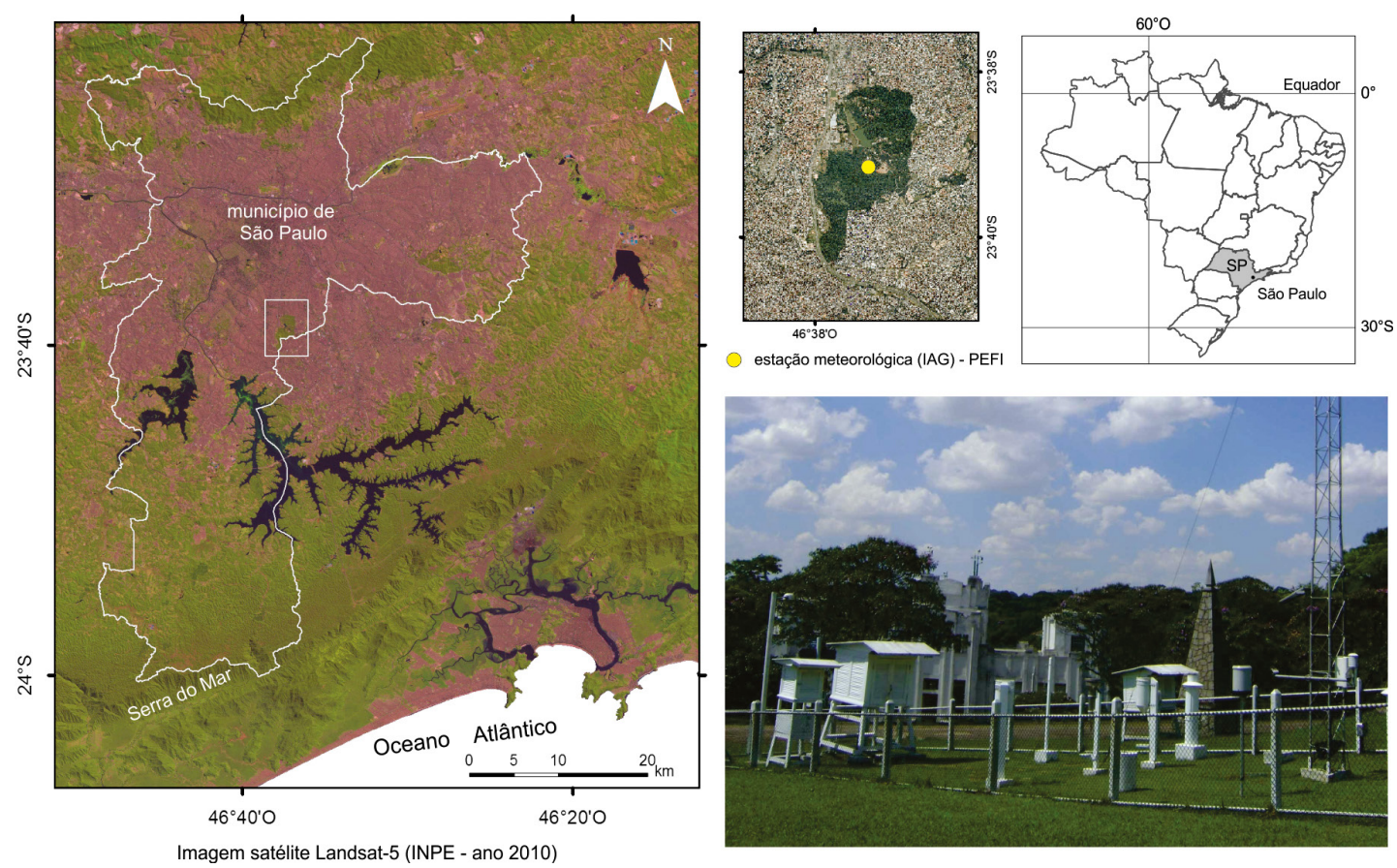

FIGURA 1 - Localização da área de estudo e detalhe do cercado meteorológico onde estão instaladas as estações meteorológicas.

\section{MATERIAIS E MÉTODOS}

3.1 Método para obtenção do fluxo de calor no solo.

O método utilizado para determinação do fluxo de calor no solo $(G)$ foi aquele desenvolvido por DECICO $(1967,1974)$ a partir da teoria analítica do calor (FOURIER 1822).

A adoção desse método baseou-se nos resultados obtidos por DECICO (1974), onde foram comparados os registros de fluxímetros e termômetros de solo. A correlação entre estes dados a cada meia hora resultou em um índice de correlação de $\mathrm{R}=0,982$.

O cálculo do fluxo de calor no solo (G), conforme proposto por DECICO (1974), é calculado por um método analítico que utiliza medidas de temperatura do solo $\left({ }^{\circ} \mathrm{C}\right)$ em duas profundidades, sendo que neste caso empregaram-se as temperaturas nas profundidades $0 \mathrm{~cm}$ e $20 \mathrm{~cm}$.

Desta forma, $G$ pode ser calculado pela equação:

$$
G=K \cdot A \cdot \frac{d T s}{d Z}[\text { Eq. 1] }
$$

Sendo,

$G=$ fluxo de calor $\left(\mathrm{W} \mathrm{m}^{-2}\right)$
$A=$ área através da qual se estuda a variação do fluxo $\left(1 \mathrm{~cm}^{2}\right)$

$\mathrm{d} T S=$ diferença de temperatura entre dois níveis $\left({ }^{\circ} \mathrm{C}\right)$

$\mathrm{d} Z=$ diferença de nível entre os sensores $(20 \mathrm{~cm})$

$K=$ condutividade térmica do solo $\left(\mathrm{W} \mathrm{m}^{-2}\right)$

Destaca-se que da $R n$ disponível à superfície do solo, uma parcela sofre o processo de condução molecular, transferindo parte da energia da superfície para as camadas mais profundas. Essa transferência de energia interna é intrínseca do material (tipo de solo) e é denominada condutividade térmica $(K)$.

A condutividade térmica do solo $(K)\left(\mathrm{W} \mathrm{m}^{-2}\right)$ é obtida pela equação:

$K=\{(10 \exp -4) .(1,275 \log \mu-0,71) .(10 \exp 1,071 \rho)\} .0,485$ [Eq. 2]

Sendo,

$\mu=$ umidade volumétrica do solo $(8,27 \%)$

$\rho=$ densidade do solo $\left(1,14 \mathrm{~g} \mathrm{~cm}^{-3}\right)$

3.1.1 Aquisição de parâmetros do solo

O método utilizado neste trabalho para obter o fluxo de calor no solo requer a identificação do tipo de solo e os seus parâmetros físicos. 
O solo no local da estação meteorológica é um Latossolo Vermelho-Amarelo de textura argilosa (STRUFFALDI-DE-VUONO 1985). A descrição dos horizontes do perfil do solo e das amostras (Tabela 1) foi realizada na área da EMA em setembro de 2007, quando da abertura da trincheira para a instalação dos sensores de $T s$.

As características físicas do solo para a determinação do $G$ são a umidade volumétrica $-\mu(\%)$, que é o volume de água contido em um determinado volume de solo, e a densidade do solo $-\rho\left(\mathrm{g} \mathrm{cm}^{-3}\right)$. A umidade volumétrica varia com a densidade do solo (GONÇALVES et al. 2011).

Esses dois parâmetros foram obtidos no Laboratório de Ecologia do Instituto de Botânica da Secretaria do Meio Ambiente do Estado de São Paulo, a partir das amostras coletadas do horizonte A1 (Tabela 1).

Aplicando-se $\mu$ e $\rho$, determinados em laboratório, na Eq. 2, obteve-se $K=0,02226 \mathrm{~W} \mathrm{~m}^{-2}$.

$K$ pode ser considerada constante no espaço e no tempo desde que seja considerada em uma camada relativamente espessa que contenha todos os constituintes do solo (DECICO 1967).

\subsection{Variáveis meteorológicas}

No interior do PEFI, o Instituto Astronômico, Geofísico e de Ciências Atmosféricas da Universidade de São Paulo (IAG-USP) mantém desde 1933 uma Estação Meteorológica Convencional (EMC) (WMO 83004), cujas medições e observações meteorológicas são realizadas a cada hora de forma ininterrupta e sem falhas até o presente. A partir de 2007, o Laboratório de Hidrometeorologia (LABHIDRO) do IAG-USP instalou no cercado meteorológico uma Estação Meteorológica Automática (EMA), que está em operação até hoje com medições a intervalos de 5 minutos. As variáveis medidas pelas estações estão de acordo com os protocolos da Organização Meteorológica Mundial (OMM) e, desta forma, as séries de dados meteorológicos do IAG-USP no PEFI possuem elevado padrão de qualidade para o período de 83 anos. Ressalta-se que a qualidade dos dados medidos pela EMA-IAG foi atestada pela comparação com os respectivos dados da EMC-IAG por FUNARI \& PEREIRA FILHO (2010).

As variáveis $R n$ e $T s$ são medidas pela EMA a cada 5 minutos e foram reduzidas para médias horárias. Foram utilizados dados de $T s$ da superfície $(0 \mathrm{~cm})$ e a $20 \mathrm{~cm}$ de profundidade. A tabela 2 apresenta as características dos sensores utilizados.

As análises foram realizadas no intervalo horário entre 8 e $16 \mathrm{~h}$, horário local. No inverno o horário final foi antecipado para as $15 \mathrm{~h}$ devido à baixa elevação solar, pois a altura do disco solar no horizonte de São Paulo, em junho/julho, é da ordem de $15^{\circ}$ a $16^{\circ}$. Essa restrição de horários, no nascente e poente, é sugerida por PEREZ et al. (1999) para se evitar possíveis inconsistências em $R n$.

O período do estudo foi de março de 2011 a fevereiro de 2016. Selecionaram-se preferencialmente as medições obtidas na ausência de precipitação durante o dia e nos dias anteriores e com insolação relativa (razão de insolação) acima de 0,60 $(n / \mathrm{N})$ obtidos da EMC, conforme proposto por PEREZ et al. (1999). A tabela 3 mostra o número de dias utilizados para os cálculos dos valores de $G$, agrupados por estações do ano.

TABELA 1 - Características do solo.

\begin{tabular}{cccccc}
\hline Horizonte & Espessura & Cor & Detalhes & $\begin{array}{c}\text { Umidade volumétrica } \\
(\mu)(\%)\end{array}$ & $\begin{array}{c}\text { Densidade }(\rho) \\
\left(\mathrm{g} \mathrm{cm}^{-3}\right)\end{array}$ \\
\hline \multirow{2}{*}{$\mathrm{A} 1$} & $25 \mathrm{~cm}$ & Marrom escuro & Raízes abundantes & 8,27 & 1,14 \\
\hline
\end{tabular}

TABELA 2 - Variáveis meteorológicas e seus respectivos sensores.

\begin{tabular}{cccccc}
\hline Variável & Sensor & Modelo & Fabricante & $\begin{array}{c}\text { Exatidão } \\
\text { (accuracy) }\end{array}$ & Unidade \\
\hline Radiação líquida $(R n)$ & Termopilha & NR-LITE-L & Kipp Zonen & $5 \%$ & $\mathrm{~W} \mathrm{~m}^{-2}$ \\
\hline Temperatura do solo $(T S)$ & Termistor & $107 \mathrm{LC}$ & Vaisala & 0,2 & ${ }^{\circ} \mathrm{C}$ \\
\hline
\end{tabular}


Foram escolhidos dias representativos das quatro estações do ano, sendo que o critério para classificar um dia como representativo, é que o valor de $G$ observado seja próximo da média de todos os dias de uma determinada estação do ano.

TABELA 3 - Número de dias por estação do ano utilizados.

\begin{tabular}{ccc}
\hline Estação & Período dos dados & $\begin{array}{c}N^{o} \text { de } \\
\text { dias }\end{array}$ \\
\hline Outono & março 2011/maio 2014 & 44 \\
Inverno & julho 2011/agosto 2014 & 41 \\
Primavera & outubro 2011/novembro 2015 & 36 \\
Verão & dezembro 2011/fevereiro 2016 & 29 \\
\hline Total & & 150 \\
\hline
\end{tabular}

\section{RESULTADOS E DISCUSSÃO}

O valor do fluxo de calor no solo $(G)$, durante o período de março de 2011 a fevereiro de 2016, totalizando 150 dias, apresentou valores máximos diários na primavera e no verão $(10,63$ $\mathrm{W} \mathrm{m}^{-2}$ e $9,27 \mathrm{~W} \mathrm{~m}^{-2}$, respectivamente); já no outono e inverno, os máximos diários foram de 6,96 $\mathrm{W} \mathrm{m}{ }^{-2}$ e $6,95 \mathrm{~W} \mathrm{~m}^{-2}$, respectivamente. Os valores mínimos diários foram da ordem de $1,56 \mathrm{~W} \mathrm{~m}^{-2}$ para outono e inverno, $1,26 \mathrm{~W} \mathrm{~m}^{-2}$ na primavera e $0,96 \mathrm{~W} \mathrm{~m}^{-2}$ no verão.

A tabela 4 mostra os resultados médios de $G$ por estação do ano, além das médias de $R n$, $T S$ e a porcentagem do $G$ em relação ao total de $R n$ $(G / R n)$, que representa a fração da energia líquida disponível no ambiente para aquecimento do solo. O inverno é a estação do ano em que, na média, a relação do fluxo de calor com a energia líquida $(G /$ $R n)$ é maior (5,93\%), e o verão a menor $(2,82 \%)$. Tanto o outono, como a primavera, situam-se próximos da média anual, com $3,28 \%$ e $2,64 \%$ respectivamente (Tabela 4).
O fluxo de calor no solo $(G)$ e a razão $G / R n$ (\%) variaram sazonalmente entre 2011 e 2016 . O verão apresentou valores de $G / R n$ em torno de $20 \%$ daquele observado no inverno, em virtude desta última ser a estação menos úmida do ano. Isto se justifica porque a parte de $R n$ que deveria ser consumida na evapotranspiração é utilizada para aquecer o solo e o ar atmosférico. No verão, $G$ tende a ser menor devido à maior nebulosidade e maior precipitação inerentes à esta estação (Figura 2). Na primavera e outono $G / R n$ são mais próximos da média anual (2,83\%).

A variação horária de $G$ apresenta a característica de mudar ao longo do período diurno, acompanhando a variação da elevação solar, enquanto $G / R n$ aumenta ao longo do período diurno, mesmo com a elevação solar diminuindo no final da tarde. Isto se justifica pela conservação de energia no solo (Figuras 2 e 3).

Valores de $G / R n$ obtidos por experimentos em áreas de Mata Atlântica e de Floresta Amazônica com a aplicação de fluxímetros, satélites e termômetros de solo obtidos são apresentados na tabela 5 , onde estão também relacionados experimentos que incluem culturas agrícolas em diversas regiões do Brasil, inclusive com características climáticas diferentes.

No semi-árido do Nordeste, puderam-se constatar valores mais elevados de G/Rn: 9,2\% em Apodi - RN (cultura de algodão em solo franco argiloso), $10,35 \%$ na Bacia do Rio Salitre - BA (área de caatinga florestada) e 14,5\% em São José do Sabuí - PB (cultura de algodão em região de transição cerrado/ caatinga). Os dois últimos valores foram obtidos por algoritmos de imagens de satélite LANDSAT. Os outros valores relacionados na tabela foram obtidos por fluxímetros. Os valores mais elevados para $G \mathrm{e}$ $G / R n$ no clima semi-árido em relação àqueles obtidos nos climas úmidos da Mata Atlântica e Floresta Amazônica justificam-se porque a parte de $R n$ que deveria ser utilizada na evapotranspiração é utilizada para aquecer o solo e o ar atmosférico.

TABELA 4 - Valores médios por estação do ano de $T s, R n, G$, e $G / R n$.

\begin{tabular}{ccccccccccc}
\hline & $\begin{array}{c}n^{o} \\
\text { dias }\end{array}$ & $\begin{array}{c}T_{s_{0 c m}} \\
\left({ }^{\circ} \mathrm{C}\right)\end{array}$ & $\begin{array}{c}\sigma \\
\left(T s_{0 c m}\right) \\
\left({ }^{\circ} \mathrm{C}\right)\end{array}$ & $\begin{array}{c}T_{20 c m} \\
\left({ }^{\circ} \mathrm{C}\right)\end{array}$ & $\begin{array}{c}\sigma \\
\left(T s_{20 \mathrm{~cm}}\right) \\
\left({ }^{\circ} \mathrm{C}\right)\end{array}$ & $\begin{array}{c}R n \\
\left(W m^{-2}\right)\end{array}$ & $\begin{array}{c}\sigma(R n) \\
\left(W m^{-2}\right)\end{array}$ & $\begin{array}{c}G \\
\left(W m^{-2}\right)\end{array}$ & $\begin{array}{c}\sigma(G) \\
\left(W m^{-2}\right)\end{array}$ & $\begin{array}{c}G / R n \\
(\%)\end{array}$ \\
\hline Outono & 44 & 28,0 & 3,0 & 26,8 & 5,2 & 103,37 & 29,32 & 3,39 & 1,42 & 3,28 \\
Inverno & 41 & 26,6 & 3,2 & 18,4 & 1,2 & 79,06 & 10,59 & 4,69 & 1,60 & 5,93 \\
Primavera & 36 & 31,3 & 3,4 & 24,2 & 1,3 & 158,60 & 22,76 & 4,18 & 2,30 & 2,64 \\
Verão & 29 & 30,2 & 5,5 & 26,0 & 1,0 & 180,88 & 25,63 & 2,49 & 2,03 & 1,38 \\
Média & 150 & 29,0 & 3,7 & 23,8 & 2,1 & 130,48 & 22,07 & 3,69 & 1,84 & 2,83 \\
\hline
\end{tabular}

$\sigma=$ desvio padrão. 

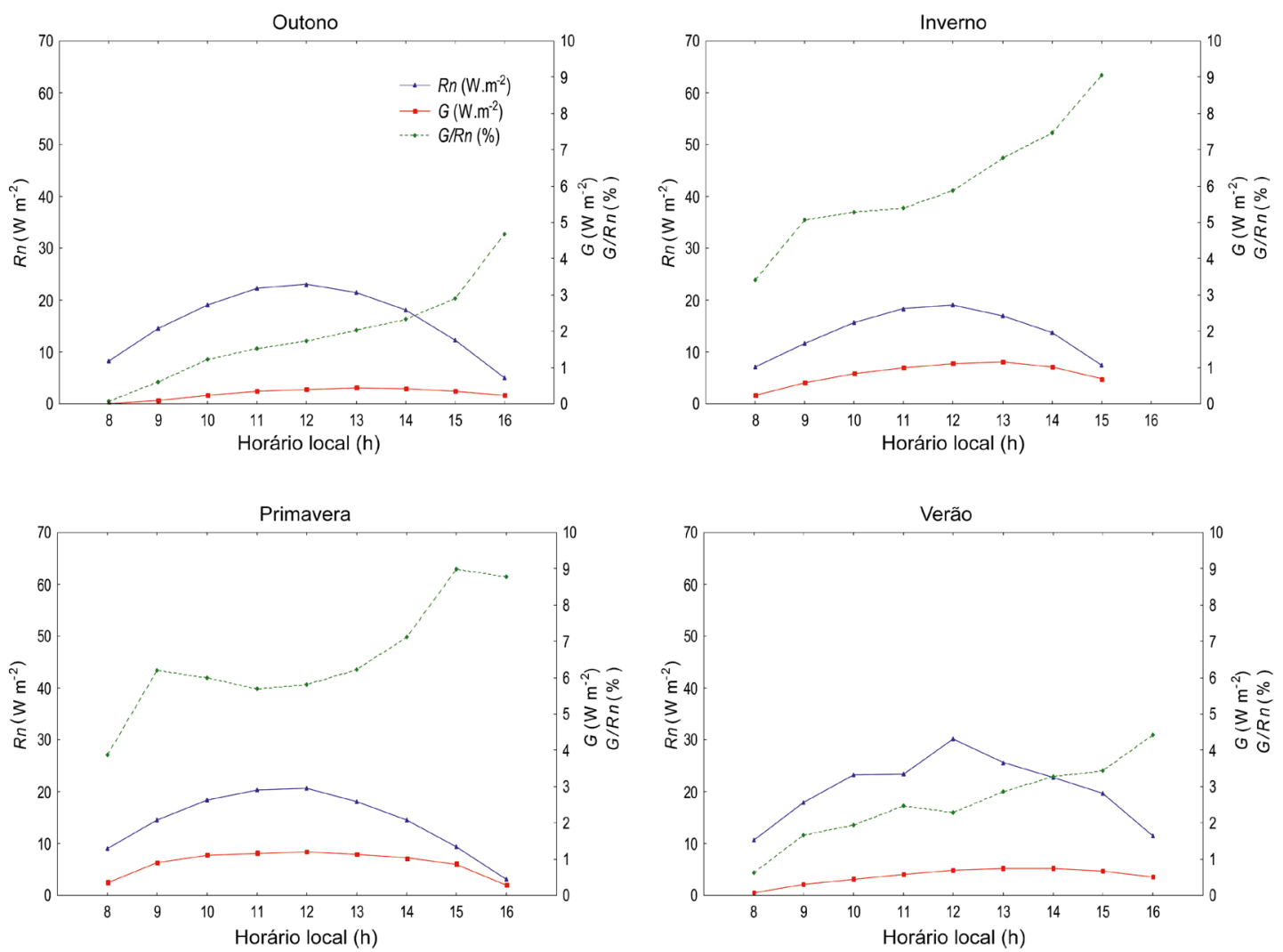

FIGURA $2-R n, G$ e $G / R n$ para dias representativos das estações do ano: Outono (05/04/2014), Inverno (16-8-2013), Primavera (10/09/2013) e Verão (26/02/2014).

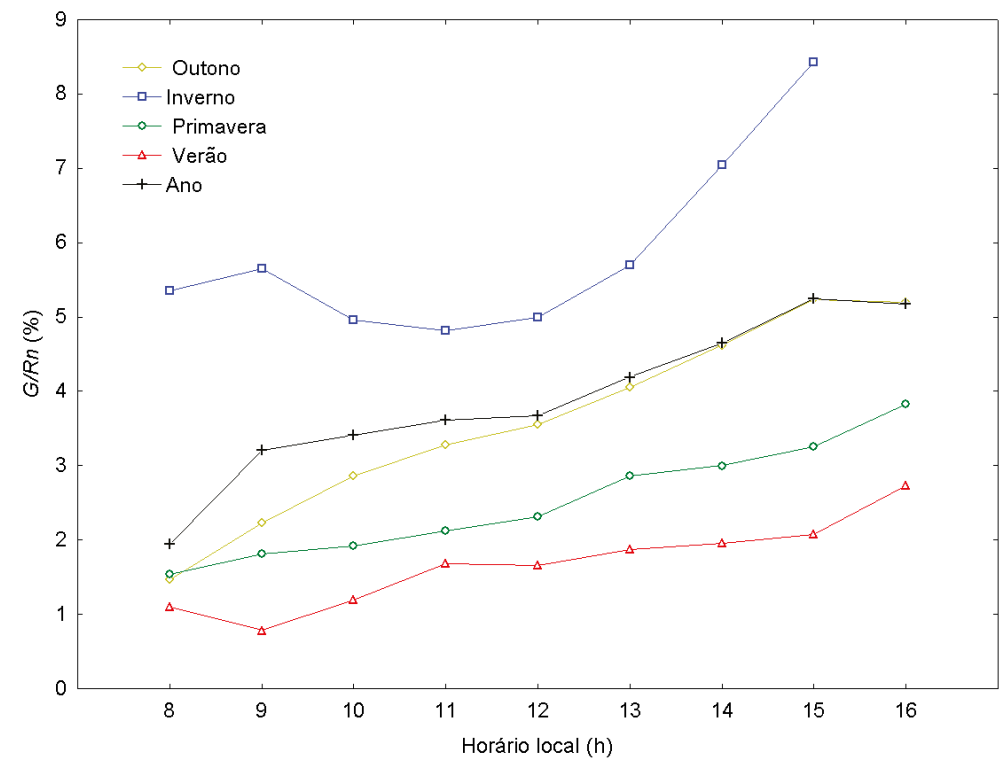

FIGURA 3 - Média horária da relação Fluxo de Calor no solo/Radiação líquida $(\mathrm{G} / \mathrm{Rn})$ para o ano e estações. 
TABELA 5 - Valores de $R n, G$ e $G / R n$ (\%) obtidos por experimentos em áreas de Mata Atlântica e de Floresta Amazônica com a aplicação de fluxímetros, satélites e termômetros de solo (termistores).

\begin{tabular}{|c|c|c|c|c|c|c|}
\hline Autor (ano) & Local & $\begin{array}{l}\text { Período / } \\
\text { Método }\end{array}$ & $\begin{array}{c}\text { Solo / Cultura ou } \\
\text { Floresta }\end{array}$ & $\begin{array}{c}R n \\
\left(W m^{-2}\right)\end{array}$ & $\begin{array}{c}G \\
\left(W m^{-2}\right)\end{array}$ & $\begin{array}{l}G / R n \\
(\%)\end{array}$ \\
\hline PINTO (2003) & $\begin{array}{l}\text { Flor. Nac. Tapajós } \\
\text { PA }\end{array}$ & $\begin{array}{c}\text { 2000/01 } \\
\text { Fluxímetros }\end{array}$ & $\begin{array}{c}\text { Latossolo amarelo } \\
\text { argiloso / Floresta } \\
\text { Amazônica }\end{array}$ & 120,52 & 1,97 & 1,63 \\
\hline LEÃO et al. (2007) & $\begin{array}{l}\text { Pilar } \\
\text { AL }\end{array}$ & $\begin{array}{c}1999 \\
\text { Fluxímetros }\end{array}$ & $\begin{array}{l}\text { Latossolo vermelho- } \\
\text { amarelo / Mata Atlântica }\end{array}$ & 156,49 & 3,13 & 2,00 \\
\hline SILVA et al. (2007) & $\begin{array}{l}\text { Piracicaba } \\
\text { SP }\end{array}$ & $\begin{array}{l}\text { 2003/2005 } \\
\text { Fluxímetros }\end{array}$ & $\begin{array}{l}\text { Latossolo argiloso / } \\
\text { Cultura de maracujá }\end{array}$ & 117,25 & 1,33 & 1,13 \\
\hline ANDRÉ et al. (2010) & $\begin{array}{c}\text { Campo Goytacases } \\
\text { RJ }\end{array}$ & $\begin{array}{c}\text { 2005/06 } \\
\text { Fluxímetros }\end{array}$ & $\begin{array}{c}\text { Cambissolo argiloso / } \\
\text { Cana de açúcar/Mata } \\
\text { Atlântica }\end{array}$ & 159,47 & 9,74 & 6,11 \\
\hline SILVA et al. (2010) & $\begin{array}{l}\text { Belterra } \\
\text { PA }\end{array}$ & $\begin{array}{c}2003 \\
\text { Fluxímetros }\end{array}$ & $\begin{array}{l}\text { Latossolo amarelo / } \\
\text { Floresta amazônica }\end{array}$ & 493,52 & 10,06 & 2,04 \\
\hline $\begin{array}{l}\text { PEREIRA et al. } \\
\text { (2011) }\end{array}$ & $\begin{array}{l}\text { Apodí } \\
\text { RN }\end{array}$ & $\begin{array}{l}2010 \\
\text { Fluxímetros }\end{array}$ & $\begin{array}{l}\text { Franco argiloso / } \\
\text { Cultura de algodão }\end{array}$ & 176,12 & 16,22 & 9,21 \\
\hline $\begin{array}{l}\text { ANDRADE } \text { et al. } \\
\text { (2009) }\end{array}$ & $\begin{array}{c}\text { Flor. Maracaí } \\
\text { MT }\end{array}$ & $\begin{array}{c}2001 \\
\text { Satélite }\end{array}$ & $\begin{array}{c}\text { Argissolo vermelho } \\
\text { amarelo / Floresta } \\
\text { Amazônica }\end{array}$ & 151,86 & 8,97 & 5,90 \\
\hline $\begin{array}{l}\text { MACHADO et al. } \\
\text { (2010) }\end{array}$ & $\begin{array}{c}\text { S.José do Sabují } \\
\text { PB }\end{array}$ & $\begin{array}{c}1998 \\
\text { Satélite }\end{array}$ & $\begin{array}{l}\text { Caatinga / cerrado } \\
\text { Cultura de algodão }\end{array}$ & 473,90 & 68,72 & 14,50 \\
\hline LOPES et al. (2011) & $\begin{array}{c}\text { Bacia R. Salitre } \\
\text { BA }\end{array}$ & $\begin{array}{c}2009 \\
\text { Satélite }\end{array}$ & $\begin{array}{l}\text { Latossolo amarelo / } \\
\text { Caatinga florestada }\end{array}$ & 748,69 & 77,50 & 10,35 \\
\hline DECICO (1974) & $\begin{array}{l}\text { Piracicaba } \\
\text { SP }\end{array}$ & $\begin{array}{c}1973 \\
\text { Fluxímetros e } \\
\text { Termistores }\end{array}$ & $\begin{array}{l}\text { Terra Roxa Estruturada / } \\
\text { Cultura de milho }\end{array}$ & 149,38 & 2,11 & 1,41 \\
\hline Este trabalho & $\begin{array}{l}\text { PEFI-CIENTEC } \\
\text { São Paulo - SP }\end{array}$ & $\begin{array}{l}\text { 2011/2016 } \\
\text { Termistores }\end{array}$ & $\begin{array}{l}\text { Latossolo vermelho- } \\
\text { amarelo / Mata Atlântica }\end{array}$ & 130,48 & 3,69 & 2,83 \\
\hline
\end{tabular}

A média de $G$ obtido no PEFI é de $3,69 \mathrm{~W} \mathrm{~m}^{-2}$, que representa $2,83 \%$ da energia líquida disponível. Esse valor é similar aos valores obtidos por DECICO (1974) e LEÃO et al. (2007) para área coberta de Mata Atlântica e são da mesma ordem de grandeza dos valores obtidos por PINTO (2003) e SILVA et al. (2010) na Floresta Amazônica.

O Parque Estadual das Fontes do Ipiranga (PEFI), reserva de Mata Atlântica preservada, está dentro da maior área urbana do Brasil, e, portanto, sofre o efeito antrópico. Conforme relatado em pesquisas anteriores, observou-se o aumento dos totais anuais de precipitação e da temperatura média e diminuição da umidade relativa do ar (PEREIRA FILHO et al. 2007), o aumento do número de dias com orvalho (ARMANI et al. 2008), bem como um conjunto de evidências sobre o estabelecimento de um ritmo antropogênico no clima da Região Metropolitana de São Paulo (RMSP) (AZEVEDO 2001). Apesar dessas constatações, a presente pesquisa pôde demonstrar apenas a variação sazonal do fluxo de calor no solo, devido à relativa brevidade do período dos dados (2011-2016).

\section{CONCLUSÕES}

A partir dos resultados obtidos no presente estudo pode-se concluir que:

1) O fluxo de calor no solo $(G)$ médio, determinado a partir do uso de medições com termômetros de solo utilizando a equação de DECICO (1967), para o PEFI é de $3,69 \mathrm{~W} \mathrm{~m}^{-2}$ ou 2,83\% da energia líquida disponível.

2) O fluxo de calor no solo apresenta variação sazonal, sendo que na média os maiores valores ocorrem no inverno $\left(4,69 \mathrm{~W} \mathrm{~m}^{-2}\right.$ ou $5,93 \%$ da energia disponível), por ser a época com menor nebulosidade. No verão há maior nebulosidade, que faz reduzir a importância de $G$ em relação a $R n(1,38 \%)$. $\mathrm{Na}$ primavera e outono os fluxos de calor no solo em relação à energia líquida disponível são mais 
próximos da média anual registrada de 2,83\% ( $2,64 \%$ e $3,28 \%$ respectivamente).

3) Este estudo contribui para o conhecimento do fluxo de calor no solo em uma área de Mata Atlântica inserida no interior de uma grande metrópole.

\section{AGRADECIMENTOS}

Agradecemos aos relatores e editores da Revista do Instituto Geológico pela discussão e sugestões valiosas para a concretização deste trabalho.

\section{REFERÊNCIAS BIBLIOGRÁFICAS}

ANDRADE, N.L.R.; AGUIA, R.G.; SANCHES, L.; ALVES, E.C.R.F.; NOGUEIRA, J.S. 2009. Partição do saldo de radiação em áreas de floresta amazônica e floresta de transição Amazônia - cerrado. Revista Brasileira de Meteorologia, 24(3): 346-355.

ANDRÉ, R.G.B.; MENDONÇA, J.C.; PINHEIRO, F.M.A.; MARQUES, V.S.; MARQUES, J. 2010. Aspectos energéticos do desenvolvimento da cana de açúcar. Revista Brasileira de Meteorologia, 25(4): 535-542.

ARMANI，G.; FUNARI，F.L.; SALUM， S.T. 2008 - Ocorrência de orvalho na cidade de São Paulo. Revista do Instituto Geológico, 29(1/2): 41-48.

AZEVEDO, T.R. 2001. Derivação antrópica do clima da Região Metropolitana de São Paulo abordada como função do ritmo semanal das atividades humanas. Faculdade de Filosofia, Letras e Ciências Humanas, Universidade de São Paulo, São Paulo, Tese de Doutorado, $473 \mathrm{p}$.

\section{IAG - INSTITUTO DE ASTRONOMIA, GEOFÍ-} SICA E CIÊNCIAS ATMOSFÉRICAS/USP. 2015. Boletim climatológico anual da estação meteorológica do IAG/USP - 2014. São Paulo: IAG/USP, 76 p. (Boletim, 17)

DECICO, A. 1967. Equações para o cálculo da condutividade térmica de algumas séries de solos. Escola Superior de Agricultura Luiz de Queiroz, Universidade de São Paulo, Piracicaba, Tese de Doutorado, 26 p.
DECICO, A. 1974. A determinação das propriedades térmicas do solo em condições de campo. Escola Superior de Agricultura Luiz de Queiroz, Universidade de São Paulo, Piracicaba, Tese de Livre Docência, 78 p.+ apêndices.

FOURIER, J.B.J. 1822. Théorie analytique de la chaleur. Paris, Chez Firmin Didot, Père et Fils, $670 \mathrm{p}$.

FUNARI，F.L. 1983. Insolação, Radiação Solar Global e Radiação Líquida no Brasil. Faculdade de Filosofia, Letras e Ciências Humanas, Universidade de São Paulo, São Paulo, Dissertação de Mestrado, 69 p. + mapas e tabelas.

FUNARI, F.L.; PEREIRA FILHO, A.J. 2010. Análise comparativa de medições de variáveis meteorológicas realizadas por estações meteorológica convencional e automática instaladas no PEFI. São Paulo-SP. Revista Brasileira de Climatologia, 6: 159-168.

FUNARI, F.L.; PEREIRA FILHO, A.J. 2014. Energy Balance in a Patch of the Atlantic Forest in São Paulo City, Brazil. Journal of Water Resource and Protection, 6: 805-812.

GALVANI, E.; ESCOBEDO, J.F.; PEREIRA, A.B. 2001 Balanço de radiação e fluxo de calor no solo em ambiente natural e protegido com pepineiro. Bragantia, 60(2): 139-147.

GONÇALVES, A.C.A.; TRINTINALHA, M.A.; TORMENA, C.A.; FOLEGATTI, M.V. 2011. Influência da densidade do solo na estimativa da umidade em um nitossolo vermelho distroférico, por meio da técnica TDR. Revista Brasileira de Ciência do solo, 35(5): 1551-1560.

LEÃO, I.B.; COSTA, G.B.; SOUZA, A.J.; BRAUNER, D.C.; TOLEDO FILHO, M.R. 2007. Balanço de energia em uma cultura de cana de açúcar no Estado de Alagoas. Ciência e Natura, Edição Especial (Micrometeorologia): 95-98.

LOPES, L.L.; TEIXEIRA, A.C.H.; ACCIOLY, L.J.O.; SOBRAL, M.C.; MONTENEGRO, S.M.G.L. 2011. Relação do fluxo de calor no solo com o uso e cobertura de terras na bacia hidrográfica do Salitre, Sertão da Bahia. In: INPE, SIMPÓSIO BRASILEIRO DE SENSORIAMENTO REMOTO, 15, Curitiba, Anais, 5785 p. 
MACHADO, C.C.C.; GALVINCIO, J.D.; OLIVEIRA, T.H. 2010. Estimativa dos valores do saldo de radiação e fluxo de calor no solo no município de São José do Sabugi - PB - usando o algoritmo SEBAL. In: SEMINÁRIO LATINO-AMERICANO DE GEOGRAFIA FÍSICA, 6, SEMINÁRIO IBERO-AMERICANO DE GEOGRAFIA FÍSICA, 2, Coimbra, Anais, 1-13.

PEREIRA, V.C.; ESPÍNOLA SOBRINHO, J.; BEZERRA, J.R.C.; MOURA, M.S.B.; BORGES, U.P.; SANTOS, W.O. 2011. Saldo de radiação e fluxo de calor no solo nas diferentes fases de da cultura do algodoeiro na chapada do Apodí-RN. In: CONGRESSO BRASILEIRO DE AGROMETEOROLOGIA, 17, Guaraparí, Anais.

PEREIRA FILHO, A.J.; MARQUES DOS SANTOS, P.; XAVIER, T.M.B.S. 2007. Evolução do Tempo e do clima na Região Metropolitana de São Paulo. Editora USP, São Paulo, 282 p.

PEREZ, P.J.; CASTELIVI, F.; IBAÑEZ, M.; ROSELL, J.I. 1999. Assessment of reliability of Bowen ratio method for partitioning fluxes. Agricultural and Forest Meteorology, 97(3): 141-150.

PINTO, L.D.V.O. 2003. Fluxos de energia sobre uma floresta tropical na Amazônia. Instituto Astronômico e Geofísico, Universidade de
São Paulo, São Paulo, Dissertação de Mestrado, $79 \mathrm{p}$.

SILVA, A.F.; PEREIRA, R.L.; MACHADO, W.B.; SILVA, R. 2010. Fluxos de energia em área agrícola e florestada na Amazônia oriental. In: UFAM, SEMINÁRIO INTERNACIONAL DE CIÊNCIAS DO AMBIENTE E SUSTENTABILIDADE, 1, Manaus, Anais, 7 p.

SILVA, T.J.A.; FOLEGATTI, M.V.; SILVA, C.R.; ALVES JR., J.; BONFIM-SILVA, E.M. 2007. Balanço de energia e estimativa da evapotranspiração em culturas irrigadas de maracujazeiro pelo método da Razão de Bowen. Engenharia Agrícola, 27(2): 392-403.

SOARES, W.A. 2013. Análise comparativa do fluxo de calor no solo em profundidade e na superfície. Revista Brasileira de Geografia Física, 6(4): 665-679.

STRUFFALDI-DE-VUONO, Y. 1985. Fitossociologia do estrato arbóreo da floresta da Reserva Biológica do Instituto de Botânica. Instituto de Biociências, Universidade de São Paulo, São Paulo, Tese de Doutorado, 213 p.

TARIFA, J.R.; ARMANI, G. 2001. Os climas 'naturais'. In: J.R. Tarifa. \& T.R. Azevedo (Ed.) Os climas na cidade de São Paulo: teoria e prática. São Paulo, GEOUSP, Coleção Novos Caminhos, 4: 47-70.

\section{Endereço dos autores:}

Frederico Luiz Funari e Augusto José Pereira Filho - Laboratório de Hidrometeorologia, Instituto de Astronomia, Geofísica e Ciências Atmosféricas, Universidade de São Paulo, Avenida Miguel Stéfano, 4200, Água Funda, CEP 04301-904, São Paulo, SP, Brasil. E-mails: ffunari@uol.com.br, augusto.pereira@iag.usp.br

Artigo submetido em 23 de fevereiro de 2017, aceito em 21 de junho de 2017. 